Chapman University

Chapman University Digital Commons

Pharmacy Faculty Articles and Research

School of Pharmacy

9-19-2013

\title{
Effects of Short-Term Portacaval Anastomosis on the Peripheral and Brain Disposition of the Blood- Brain Barrier Permeability Marker Sodium Fluorescein in Rats
}

Imam H. Shaik

Texas Tech University Health Sciences Center

M. K. Miah

Texas Tech University Health Sciences Center

Ulrich Bickel

Texas Tech University Health Sciences Center

Reza Mehvar

Chapman University, mehvar@chapman.edu

Follow this and additional works at: http://digitalcommons.chapman.edu/pharmacy_articles

Part of the Other Pharmacy and Pharmaceutical Sciences Commons, Pharmaceutical Preparations Commons, and the Pharmaceutics and Drug Design Commons

\section{Recommended Citation}

Shaik IH, Miah MK, Bickel U, and Mehvar R. Effects of Short-Term Portacaval Anastomosis on the Peripheral and Brain Disposition of the Blood-Brain Barrier Permeability Marker Sodium Fluorescein in Rats. Brain Res., 1531:84-93 (2013). doi: 10.1016/

j.brainres.2013.07.040

This Article is brought to you for free and open access by the School of Pharmacy at Chapman University Digital Commons. It has been accepted for inclusion in Pharmacy Faculty Articles and Research by an authorized administrator of Chapman University Digital Commons. For more information, please contact laughtin@chapman.edu. 


\section{Effects of Short-Term Portacaval Anastomosis on the Peripheral and Brain Disposition of the Blood-Brain Barrier Permeability Marker Sodium Fluorescein in Rats}

Comments

NOTICE: this is the author's version of a work that was accepted for publication in Brain Research. Changes resulting from the publishing process, such as peer review, editing, corrections, structural formatting, and other quality control mechanisms may not be reflected in this document. Changes may have been made to this work since it was submitted for publication. A definitive version was subsequently published in Brain Research, volume 1531, in 2013. DOI: 10.1016/j.brainres.2013.07.040

The Creative Commons license below applies only to this version of the article.

\section{Creative Commons License}

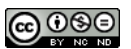

This work is licensed under a Creative Commons Attribution-Noncommercial-No Derivative Works 4.0 License.

\section{Copyright}

Elsevier 


\title{
Effects of short-term portacaval anastomosis on the peripheral and brain disposition of the blood-brain barrier permeability marker sodium fluorescein in rats
}

\author{
Imam H. Shaik', Mohammad K. Miah, Ulrich Bickel, and Reza Mehvar * \\ Department of Pharmaceutical Sciences and Center for Blood-Brain Barrier Research, School of \\ Pharmacy, Texas Tech University Health Sciences Center, Amarillo, TX 79106, United States
}

* Corresponding author: Reza Mehvar, Ph.D., School of Pharmacy, Texas Tech University Health Sciences Center, 1300 Coulter, Amarillo, TX 79106, USA; Phone: (806) 356-4015 Ext. 337; FAX: (806) 356-4034; E-mail: reza.mehvar@ttuhsc.edu.

${ }^{1}$ Present Address: Department of Pharmaceutical Sciences, University of Pittsburgh, Pittsburgh, PA 15261, United States 


\section{ABSTRACT}

Contradictory results have been reported with regard to the effects of various models of hepatic encephalopathy on the blood-brain barrier (BBB) permeability, which may be due partly to the use of brain concentrations of BBB markers without attention to their peripheral pharmacokinetics. The purpose of the current study was to investigate the effects of short-term portacaval anastomosis (PCA), a type B model of hepatic encephalopathy, on the peripheral pharmacokinetics and brain distribution of sodium fluorescein (FL), which is a small molecule marker of BBB passive permeability. A single $25 \mathrm{mg} / \mathrm{kg}$ dose of FL was administered intravenously to 10-day PCA and sham-operated rats, and serial blood and bile (0-30 min) and terminal (30 min) brain samples were collected, and the concentrations of FL and its glucuronidated metabolite (FL-Glu) were measured by HPLC. Additionally, the free fractions of FL $\left(f_{u}\right)$ in all the plasma samples were determined, and the effects of bile salts on $f_{u}$ were investigated in vitro. Passive permeability of BBB to FL was estimated by brain uptake clearance $\left(K_{i n}\right)$ based on both the brain concentrations of FL and plasma concentrations of free (unbound) FL. PCA caused a $26 \%$ increase in the $f_{u}$ of FL in plasma, which was due to competition of bile acids with FL for binding to plasma proteins. Additionally, PCA reduced the biliary excretion of FL-Glu by $55 \%$. However, free $K_{\text {in }}$ values $(\mu 1 / \mathrm{min} / \mathrm{g}$ brain) for the sham $(0.265 \pm 0.034)$ and PCA $(0.228 \pm 0.038)$ rats were not significantly different. It is concluded that whereas 10-day PCA alters the peripheral pharmacokinetics of FL, it does not significantly affect the BBB permeability to the marker.

Keywords: Blood-brain barrier; Sodium fluorescein; Brain uptake clearance; Plasma free fraction; Portacaval anastomosis; Hepatic encephalopathy 


\section{Introduction}

Hepatic encephalopathy (HE) is a neuropsychiatric disorder associated with hepatocellular failure or portal-systemic venous shunting, which results in exposure of the brain to high concentrations of toxins that are otherwise removed by the liver. In addition to significant effects on the brain function, it has been suggested that HE may also increase the blood-brain barrier (BBB) permeability in different models of HE (Cauli et al., 2011; Chen et al., 2013; Dixit and Chang, 1990; Horowitz et al., 1983; Laursen and Westergaard, 1977; Livingstone et al., 1977; Nguyen, 2012; Shimojima et al., 2008; Zaki et al., 1984). However, other studies have shown that HE does not affect the BBB passive permeability (Alexander et al., 2000; Bémeur et al., 2010; Bosoi et al., 2012; Jin et al., 2013). One obvious explanation for these apparently contradictory results is the heterogeneity of the animal models of HE used in these studies. According to the latest definitions (Ferenci et al., 2002), HE is divided into three types based on the extent and type of liver injury that are associated with acute liver failure (type A), portalsystemic bypass (type B), and cirrhosis (type C). Therefore, it is likely that different types of HE have different effects on the BBB permeability.

A second complicating factor in the reported contradictory results may be the methodology to determine the BBB permeability. For example, in some reports (Bosoi et al., 2012; Chen et al., 2013; Shimojima et al., 2008) sodium fluorescein (FL) is used as a small molecule, non-permeable marker to study the passive permeability of the BBB. Generally, these studies use the brain concentration of FL as a measure of BBB integrity. However, HE may also alter the peripheral pharmacokinetics and area under the plasma concentration-time curve (AUC) of FL, thus potentially affecting the brain concentrations of the marker even in the absence of any changes in the BBB passive permeability. Additionally, FL is bound to proteins in the 
plasma (Li and Rockey, 1982; Manzini and Crescenzi, 1979), and only the free (unbound) fraction of the drug is available for distribution to other organs (Mehvar, 2005), including the brain. Therefore, studies using FL as an in vivo marker of BBB permeability in HE should consider the effects of the disease on both the extent of systemic exposure (AUC) and free fraction of the drug, in addition to its brain concentrations.

Rats with portacaval anastomosis (PCA) are considered one of the best models of latent encephalopathy (type B) in humans (Butterworth et al., 2009). Additionally, few studies have reported the effects of PCA in rats on the integrity of the BBB (Alexander et al., 2000; Bosoi et al., 2012; Laursen and Westergaard, 1977; Sumner, 1982). Whereas earlier studies (Laursen and Westergaard, 1977; Sumner, 1982) suggested that the BBB permeability to the macromolecule horseradish peroxidase (HRP) is increased at 10, 14, or 30 days after PCA, more recent studies (Alexander et al., 2000; Bosoi et al., 2012) using mannitol, Evans blue, or FL after 4 or 16 weeks of PCA reported no effects of PCA on the BBB passive permeability. The disagreement among these studies may be related, at least in part, to the time course of the PCA effects on the BBB permeability and/or the size and mechanism of the passage of the marker through the BBB. However, we are not aware of any quantitative studies on the short-term ( $\leq 14$ days) effects of PCA on the BBB permeability of small, non-permeable markers, such as FL. Therefore, the purpose of the current investigation was to study the effects of short-term (10 day) PCA on the BBB passive permeability using a quantitative analysis of the peripheral and brain disposition of FL, including consideration of its free fraction in plasma $\left(f_{u}\right)$. Based on the reported increase in the BBB to HRP at 10-14 days after PCA (Laursen and Westergaard, 1977; Sumner, 1982), we hypothesized that short-term PCA increases the BBB passive permeability to FL. 


\section{Results}

The liver weight (as a fraction of total body weight) and plasma biochemical parameters for the Sham and PCA rats are shown in Fig. 1. PCA caused a $16 \%$ decrease $(p<0.05)$ in the liver: total body weight ratio (Fig. 1a). Additionally, PCA caused a $40 \%$ increase $(p<0.05)$ in the plasma concentration of ammonia (Fig. 1b) and an almost 7-fold increase $(p<0.0001)$ in the concentrations of total bile acids in plasma (Fig. 1c). Although PCA also modestly (11\%) reduced $(p<0.01)$ the total plasma protein concentrations (Fig. 1d), the plasma albumin concentration was not affected by the surgery (Fig. 1e). Furthermore, the plasma concentrations of aspartate aminotransferase (AST) in the Sham and PCA animals were not significantly different (Fig. 1f).

The total (free plus bound) and free plasma concentration-time courses of FL, along with $f_{u}$ values of the marker, are presented in Fig. 2. Additionally, the zero to 30 min AUC values of total $\left(A U C_{\text {total }}\right)$ and free $\left(A U C_{\text {free }}\right)$ FL during the sampling time are presented in Table 1. Although the plasma concentrations or AUC values for the total or free FL were not significantly different in the PCA and Sham groups, the $A U C_{\text {free }} / A U C_{\text {total }}$ ratio in the PCA animals was $26 \%$ higher $(p<0.01)$ than that in the Sham group (Table 1). This was due to significantly higher $f_{u}$ of FL in the PCA animals in most of the samples (Fig. 2c), which also resulted in reversal of PCA: Sham AUC ratios when total (ratio of 0.903) and free (ratio of 1.15) AUCs were considered (Table 1).

The biliary excretion data for FL and its glucuronidated metabolite (FL-Glu) in the Sham and PCA rats are depicted in Fig. 3. Although PCA did not significantly affect the biliary recovery (Fig. 3a) or biliary clearance of total (Fig. 3b) or free (Fig. 3c) FL, it significantly $(p<0.01)$ reduced the biliary excretion of FL-Glu from $17.6 \%$ of the dose to $7.93 \%$ of the dose 
(Fig. 3d). Consequently, the overall (FL plus FL-Glu) biliary recovery in the PCA rats (17.9\% of dose) was significantly $(p<0.05)$ lower than that in the Sham animals $(28.7 \%$ of dose $)$ (Fig. 3e). Further, the bile flow rate $(\mathrm{ml} / \mathrm{h})$ in the PCA animals $(1.09 \pm 0.27)$ was significantly $(p<0.01)$ lower than that in the Sham group $(1.74 \pm 0.27)$ (Fig 3f).

The concentration and amount of FL in the liver of Sham and PCA rats are depicted in Fig. 4. Whereas the liver concentrations of FL in the Sham and PCA rats were not significantly different from each other (Fig. 4a), the amount of FL recovered in the liver of PCA rats (5.89\% $\pm 0.93 \%$ of dose) was significantly $(p<0.05)$ lower than that in the Sham animals $(7.74 \%$ $\pm 1.33 \%$ of dose) (Fig. 4 b), most likely due to the lower weight of the liver in the PCA rats. FLGlu was not detectable in the liver samples.

The brain concentration and total (free plus bound) and free brain uptake clearance $\left(K_{i n}\right)$ values for FL in the Sham and PCA rats are shown in Fig. 5. PCA did not significantly affect the brain concentration (Fig. 5a) or the total (Fig. 5b) or free (Fig, 5c) $K_{\text {in }}$ values of FL. However, although the PCA: Sham ratio was more than 1 for the total $K_{\text {in }}(1.08)$, the ratio was less than 1 (0.857) for the free $K_{\text {in }}$ (Fig. 3).

The effects of different concentrations of major bile acids on the in vitro $f_{u}$ of FL are demonstrated in Fig. 6. All the tested bile acids caused a concentration-dependent increase in the $f_{u}$ of FL. Compared with the baseline $f_{u}$ value of 0.264 , the highest concentration of bile acids $\left(150 \mu \mathrm{M}\right.$ ) resulted in $f_{u}$ values of $0.414-0.433$ (Fig. 6), representing $57 \%$ to $64 \%$ increase in the $f_{u}$.

\section{Discussion}

The main goal of the current study was to investigate the effects of short-term (10 day) PCA on the BBB passive permeability to the small molecule probe FL using an appropriate kinetic 
analysis, considering both the peripheral and brain disposition of the marker. The success of PCA operation was confirmed by reductions in the liver: total body weight ratio and substantial increases in the plasma concentrations of ammonia and total bile acids (Fig. 1), in addition to visual inspection of the shunt at the time of liver collection. These observations are in agreement with previous reports on the effects of PCA on the liver: total body weight ratio (Balasubramaniam et al., 1976; Magide et al., 1976), ammonia (Alexander et al., 2000; Hawkins et al., 1996), and total bile acids (Poupon et al., 1977).

PCA caused significant changes to the peripheral pharmacokinetics of FL, most notably an increase in the $f_{u}$ of the marker (Fig. 2c) and a decrease in the biliary excretion of its glucuronidated metabolite (Fig. 3d). As for protein binding, the $f_{u}$ of FL in both Sham and PCA rats was significantly $(p<0.0001)$ affected by the sampling time (Fig. $2 c)$, suggesting nonlinear, concentration-dependent binding of the marker to plasma proteins; the $f_{u}$ values at earlier time points (higher plasma concentrations) were higher than those at later time points (lower plasma concentrations). The concentration-dependent $f_{u}$ of FL, observed in our studies, is in agreement with a previous study (Grimes, 1985) that reported $f_{u}$ values of $0.496,0.212$, and 0.074 in Wistar rats at FL plasma concentrations of 446, 163, and $25 \mu \mathrm{g} / \mathrm{ml}$, respectively.

Various studies were conducted to determine the possible mechanisms of the PCAinduced increase in the $f_{u}$ of FL. A PCA-induced decrease in the total plasma proteins (Fig. 1d) might have potentially been responsible for the decrease in the $f_{u}$ of FL. However, being an acid, FL is expected to bind almost exclusively to plasma albumin (Manzini and Crescenzi, 1979; Rockey et al., 1983), which was not significantly affected by PCA (Fig. 1e). In contrast, the most striking effect of PCA on biochemical parameters in our study was the 7-fold increase in the plasma concentrations of total bile acids (Fig. 1c). Bile acids are known to bind significantly to 
albumin (most likely site I) (Roda et al., 1982), displacing other drugs, such as warfarin (Bowmer et al., 1985), from their binding site. FL is reportedly bound to albumin site I because warfarin directly competes with fluorescein for the same binding site on the serum albumin (Manzini et al., 1979). Therefore, we hypothesized that the PCA-induced increase in the $f_{u}$ of FL was due to the 7-fold higher concentrations of total bile acids in plasma. Indeed, in vitro studies with four major bile acids in plasma are in agreement with this hypothesis as all the tested bile acids concentration-dependently increased the $f_{u}$ of fluorescein in plasma (Fig. 6).

The second major change in the peripheral pharmacokinetics of FL was the substantial reductions in the biliary excretion of FL-Glu (Fig. 3d). Although we are not aware of any specific reports on the mode of transport of FL-Glu into the bile, the biliary transport of FL is through Mrp2 (Mills et al., 1999). Because Mrp2 is a major transporter for glucuronidated compounds, it is likely that the transport of FL-Glu, similar to its parent drug, is also mediated by Mrp2. Theoretically, the observed reduction in the biliary excretion of FL-Glu in the PCA rats (Fig. 3d) could be due to a decreased formation and/or reduced biliary transport of the metabolite. Because PCA did not significantly affect the Mrp2-dependent biliary clearance of FL (Figs 3b and c), it is likely that the substantial decrease in the biliary recovery of FL-Glu is due to its reduced formation, rather than a reduced transport.

The quantitative analysis of brain uptake, incorporating both the brain concentrations and AUC values of the total or free FL suggested that 10-day PCA in our rat model does not significantly alter the BBB permeability to the marker (Fig. 5). Similarly, Bosoi et al. (Bosoi et al., 2012) recently showed that there was no difference between the Sham and PCA rats in their brain concentrations of Evans blue or FL at 4 weeks after the surgery. Although the Mw of Evans blue is $961 \mathrm{Da}$, it avidly binds to albumin in plasma, forming a macromolecule. Therefore, 
that study (Bosoi et al., 2012) suggests that the permeability of the BBB to both small molecules (FL) and macromolecules (Evans blue) remain unchanged in the 4 week-PCA rats. Additionally, Alexander et al. (Alexander et al., 2000) reported no effect of PCA on the brain ${ }^{14}$ C-mannitol space in 16 week PCA rats using the brain in situ perfusion method; mannitol is a non proteinbound, non-metabolizable probe for BBB passive permeability. These observations are in contrast to histological experiments that showed an increase in the permeability of the BBB vasculature to HRP at 10 or 30 days (Laursen and Westergaard, 1977) or at 14 days (Sumner, 1982) after PCA in rats. Although these earlier studies suggested a generalized increase in the BBB permeability, the more recent works by others (Alexander et al., 2000; Bosoi et al., 2012) and our own current data suggest that the apparent PCA-induced increase in HRP uptake by brain vasculature does not translate into measurable quantitative changes in the $\mathrm{BBB}$ permeability to small (FL and mannitol) or large (Evans blue) molecules. Therefore, the reported increase in the uptake of HRP by cerebral endothelium of PCA rats (Laursen and Westergaard, 1977; Sumner, 1982) may be a unique phenomenon only affecting the vesicular transport of HRP rather than a breakdown of $\mathrm{BBB}$.

Although the same conclusion (i.e., no change in BBB permeability to FL) is made in our study based on the total (Fig. 5b) and free (Fig. 5c) $K_{\text {in }}$ values, this observation warrants some comments. When the total $K_{\text {in }}$ is considered, the PCA: Sham ratio is slightly more than 1 (1.08) (Fig. 5b). However, for free $K_{i n}$, the ratio is slightly less than 1 (0.857) (Fig. 5c). Therefore, it is conceivable that under a different scenario, such as PCA: Sham ratio of 1 or slightly lower than 1 for the total $K_{i n}$, the differences between the PCA and Sham for free $K_{i n}$ would have been statistically significant, hence resulting in a different conclusion based on the total and free $K_{i n}$. 
Consequently, although not exemplified in our studies, it seems prudent to use free $K_{\text {in }}$ when FL is used as a marker for the in vivo determination of BBB passive permeability.

The in vivo approach used here for determination of BBB permeability using FL has been used before for ${ }^{14} \mathrm{C}$-sucrose (Bickel et al., 1998; Kang et al., 1994; Ohno et al., 1978), which is the gold standard for determination of BBB passive permeability to small molecules (Mw of 342 Da). The reported in vivo $K_{\text {in }}$ for ${ }^{14} \mathrm{C}$-sucrose, which is not bound to plasma proteins, is $0.25-0.30$ $\mu \mathrm{l} / \mathrm{min} / \mathrm{g}$ brain (Bickel et al., 1998). It is interesting to note that the free $K_{\text {in }}$ value of $0.265 \pm$ $0.034 \mu \mathrm{l} / \mathrm{min} / \mathrm{g}$ brain for FL (Mw of $332 \mathrm{Da}$ ) reported here in Sham animals (Fig. 5c) is strikingly close to the reported (Bickel et al., 1998) value for sucrose. Therefore, it appears that ${ }^{14} \mathrm{C}$-sucrose and FL have similar properties in terms of kinetics of brain uptake. However, it should be noted that in contrast to ${ }^{14} \mathrm{C}$-sucrose, some studies (Sun et al., 2001b) have suggested that Mrp1 might be involved in the brain distribution of fluorescein, although additional studies (Sun et al., 2001a) showed that the brain accumulation of fluorescein did not increase in Mrp1 knockout mice, suggesting a limited role for Mrp1 function at the BBB. Nevertheless, the possibility of contribution of transporters to the brain distribution of fluorescein cannot be ruled out at this time.

Although previous reports have indicated that ammonia (Skowronska et al., 2012; Ziylan et al., 1993) and bile salts (Greenwood et al., 1991; Spigelman et al., 1983) increase the BBB passive permeability, the PCA-induced increases in the plasma concentrations of these chemicals did not result in a change in the permeability of BBB to FL in our studies. This is most likely due to the differences in the concentrations of ammonia or bile acids achieved in our PCA rats and the concentrations of these chemicals used in previous studies. For example, an in vitro study (Skowronska et al., 2012) showed that treatment of a rat brain endothelial cell line with a $5 \mathrm{mM}$ 
concentration of ammonia for $24 \mathrm{~h}$ increased the cell permeability to dextran $40 \mathrm{kDa}$. Additionally, injection of ammonium acetate to rats, producing a plasma concentration of 1.45 $\mathrm{mM}$ of ammonia, resulted in BBB breakdown (Ziylan et al., 1993). However, the plasma ammonia concentrations in our PCA rats $(247 \pm 47 \mu \mathrm{M})$, although significantly higher than those in the Sham animals $(176 \pm 49 \mu \mathrm{M})$, were much lower than the in vitro and in vivo concentrations of ammonia that reportedly (Skowronska et al., 2012; Ziylan et al., 1993) alter BBB permeability. Similarly, using an in situ brain perfusion model, the increase in the BBB permeability to deoxycholate and taurochenodeoxycholate occurred only at perfusate concentrations of $\geq 1 \mathrm{mM}$ and $\geq 0.2 \mathrm{mM}$ (Greenwood et al., 1991), respectively. However, the total bile acid concentrations in our PCA rats were $\leq 100 \mu \mathrm{M}$ (Fig. 1c). Indeed, another study (Zaki et al., 1983) showed that BBB permeability to inulin remained unchanged by intravenous administration of glycocholic acid or taurocholic acid to rats, producing plasma concentrations of $80 \mu \mathrm{M}$. Therefore, the lack of change in the BBB permeability in our PCA rats, despite the observed increases in the plasma concentrations of ammonia and total bile acids, is in agreement with the literature data.

In conclusion, the effects of short-term (10 days $)$ PCA on the peripheral pharmacokinetics and brain distribution of the BBB passive permeability marker sodium fluorescein were investigated in rats. PCA caused a significant increase in the plasma free fraction of the marker, most likely due to an increase in the plasma concentrations of bile acids. Additionally, PCA significantly reduced the biliary excretion of the glucuronidated metabolite of the marker. However, quantitative analysis of the brain uptake of the marker indicated that the 10-day PCA does not significantly alter the BBB passive permeability. 


\section{Experimental procedures}

\section{1. Chemicals}

Sodium fluorescein (FL), $\beta$-glucuronidase (Type-LII), cholic acid (CA), chenodeoxycholic acid (CDCA), lithocholic acid (LCA), and taurocholic acid (TCA) were purchased from SigmaAldrich (St. Louis, MO). Ketamine and xylazine solutions for anesthesia were purchased from Llyod Laboratories (Shenandoah, IA). The kits for measurement of biochemical parameters were obtained from the following sources: total protein from Pierce Biotechnologies (Rockford, IL), albumin from Active Motif (Carlsbad, CA), total bile acids (TBA) from Crystal Chem (Downers Grove, IL), ammonia from Sigma-Aldrich, and aspartate aminotransferase (AST) from Teco Diagnostics (Anaheim, CA). Methanol and PIC-A reagent (tetrabutylammonium dihydrogen phosphate) for HPLC were from Fisher Scientific (Fair Lawn, NJ) and Waters (Milford, MA), respectively.

\subsection{Animals}

Adult, male Sprague-Dawley rats were obtained from Charles River Laboratories, Inc. (Wilmington, MA, USA) and kept in a temperature- and humidity-controlled room with free access to food and water before the experiments. All the animal experiments were approved by our Institutional Animal Care and Use Committee.

\subsection{Portacaval anastomosis surgery}

End-to-side portacaval anastomosis (PCA) was carried out at Charles River Laboratories. Briefly, the procedure consisted of clamping of the portal vein and inferior vena cava of isofluraneanesthetized animals for $\sim 5 \mathrm{~min}$, followed by end-to-side anastomosis of the portal vein to inferior vena cava using a cyanoacrylate skin adhesive glue (Coy et al., 1991; Jerkins and Steele, 
1988). However, the blood flow to the liver via hepatic artery was kept intact. Sham rats underwent anesthesia, laparatomy, and 5 min clamping of the portal vein and inferior vena cava without the anastomosis. Following the surgery, animals had free access to food and water and were used in the pharmacokinetic study 10 days later.

\subsection{Pharmacokinetic study}

Ten days after PCA or sham-operation, rats were anesthetized using an intramuscular injection of ketamine: xylazine $(100: 8 \mathrm{mg} / \mathrm{kg}$ ), and catheters were placed in femoral artery (blood sampling) and bile duct (bile collection). Subsequently, fluorescein sodium at a dose of $25 \mathrm{mg} / \mathrm{kg}$ (acid equivalent) was infused into the penile vein over $5 \mathrm{~min}$. Blood samples $(\sim 250 \mu 1)$ were then collected into heparinized microcentrifuge tubes at time zero (before the drug infusion), 5, 10, 20, and $30 \mathrm{~min}$, and plasma was separated. Cumulative (0-30 min) bile samples were also collected in pre-weighted microcentrifuge tubes. At the end of experiments $(30 \mathrm{~min})$, the vasculature blood was flushed out with $50 \mathrm{ml}$ of ice-cold saline delivered at a flow rate of $25 \mathrm{ml} / \mathrm{min}$ through a catheter inserted into the left ventricle of the heart, and brain and liver tissue were collected. The brain and liver were snap-frozen in dry ice/isopentane and liquid nitrogen, respectively. All the samples were stored at $-80^{\circ} \mathrm{C}$ until analysis.

\subsection{Protein binding studies}

The unbound concentrations of fluorescein in plasma were determined by ultrafiltration method using Microcon Ultracel YM-30 centrifugal devices, with a molecular weight cut-off of $30 \mathrm{kDa}$, from Millipore (Billerica, MA, USA). Approximately, $100 \mu \mathrm{l}$ of each plasma sample was added to the device, which was then incubated at $37^{\circ} \mathrm{C}$ for $30 \mathrm{~min}$ before centrifugation at $2000 \mathrm{~g}$ for 15 $\min \left(37^{\circ} \mathrm{C}\right)$. Aliquots $(10 \mu \mathrm{l})$ of the original plasma samples and the filtrate were then subjected 
to the HPLC assay described below. The free fraction $\left(f_{u}\right)$ of fluorescein was estimated by dividing the concentration of the marker in the filtrate (free) by that in the original plasma sample (total).

\subsection{Effects of bile salts on unbound fraction of fluorescein in plasma}

The effects of various bile acids on the $f_{u}$ of FL in plasma were investigated in an in vitro study. Drug-free, heparinized rat plasma (Innovative Research, Novi, MI) was spiked with LCA, CA, CDCA, or TCA at concentrations of $0,18.75,37.5,75$, or $150 \mu \mathrm{M}$. After addition of FL at a plasma concentration of $50 \mu \mathrm{g} / \mathrm{ml}$, the samples ( $n=3 /$ group) were incubated at $37^{\circ} \mathrm{C}$ for $30 \mathrm{~min}$ before ultrafiltration as described above.

\subsection{Fluorescence HPLC analysis of fluorescein}

The concentrations of FL in plasma, bile, liver, and brain samples were measured by modification of a previously reported HPLC method (Selan et al., 1985). Briefly, the samples were chromatographed on a reversed phase $\mathrm{C}_{18}$ column $(250$ x $4.6 \mathrm{~mm}, 5 \mu \mathrm{m}$; Microsorb-MV 100-5 $\mathrm{C}_{18}$; Varian, Palo Alto, CA) with a mobile phase of methanol: water: PIC-A reagent (55: 45: 1.5, v/v; $\mathrm{pH} 7.4$ ), run at a flow rate of $0.8 \mathrm{ml} / \mathrm{min}$. Fluorescence detection at excitation and emission wavelengths of 495 and $520 \mathrm{~nm}$, respectively, was used for quantitation of FL.

Before analysis, liver (1:9) and brain (1:4) tissues were homogenized in distilled water. Additionally, plasma, filtrate after ultrafiltration of plasma, and liver homogenate samples were diluted 100, 50, and 5 times, respectively, with a solution of $4 \%$ bovine serum albumin in water before analysis. The brain homogenate samples were used without further dilution. To $20 \mu 1$ of each sample was added $200 \mu \mathrm{l}$ of mobile phase, and the samples were vortex-mixed for 10 sec. After centrifugation, $50 \mu \mathrm{l}$ of the supernatant was injected into the HPLC system. Bile samples 
were analyzed twice, before and after hydrolysis. Glucuronide hydrolysis was based on a published method (Chen et al., 1980). Briefly, $20 \mu \mathrm{l}$ of the diluted bile sample was incubated for $20 \mathrm{~min}\left(37^{\circ} \mathrm{C}\right)$ with $50 \mu \mathrm{l}$ of a $0.25 \mathrm{M}$ sodium acetate buffer $(\mathrm{pH} 4.75)$ that contained 500 units of $\beta$-glucuronidase. Subsequently, $150 \mu \mathrm{l}$ of mobile phase was added, and after mixing and centrifugation, $50 \mu \mathrm{l}$ of the supernatant was injected into the HPLC. Preliminary studies indicated that the concentrations of FL-Glu in the plasma, liver, and brain samples were negligible or undetectable. Therefore, only the parent drug was measured in these samples.

\subsection{Analysis of biochemical parameters in plasma}

The plasma concentrations of several biochemical markers were quantitated according to the manufacturers' instructions for the commercially-available kits based on the following methods: Total plasma protein by the BCA method; albumin by albumin blue fluorescent assay; total bile acids by enzyme cycling method; ammonia by enzymatic (glutamate dehydrogenase) assay; and AST by spectrophotometric method.

\subsection{Pharmacokinetic analysis}

The areas under the plasma concentration-time curve of total $\left(A U C_{\text {total }}\right)$ and free $\left(A U C_{f r e e}\right) \mathrm{FL}$ during the sampling time $(0-30 \mathrm{~min})$ were estimated using linear trapezoidal rule. The apparent brain uptake clearances $\left(K_{\text {in }}\right)$ for the total and free FL were estimated by dividing the brain concentration of FL at $30 \mathrm{~min}$ by the $A U C_{\text {total }}$ and $A U C_{\text {free }}$, respectively (Bickel, 2005). The biliary clearance of total and free FL was estimated by dividing the amount of the marker excreted into the bile during the 30 -min sampling period by the $A U C_{\text {total }}$ and $A U C_{\text {free }}$, respectively. 


\subsection{Statistical analysis}

Except for the free fraction, the differences between the Sham and PCA for all parameters were tested using unpaired, two-tailed t-test. A repeated measure, two-way ANOVA, followed by Bonferroni's multiple comparison test was used the detection of significant differences between the free fraction of FL in Sham and PCA plasma at different times after the drug administration. In all cases, a $p<0.05$ was considered significant. Whenever mean data are presented, the error term is $\mathrm{SD}$.

\section{Acknowledgments}

The authors would like to acknowledge financial support from the Blood-Brain Barrier Research Center at Texas Tech School of Pharmacy. 


\section{REFERENCES}

Alexander, B., Li, X., Benjamin, I.S., Segal, M.B., Sherwood, R., Preston, J.E., 2000. A quantitative evaluation of the permeability of the blood brain barrier of portacaval shunted rats. Metab. Brain. Dis. 15, 93-103.

Balasubramaniam, S., Press, C.M., Mitropoulos, K.A., Magide, A.A., Myant, N.B., 1976. Effect of portacaval anastomosis on the activities of hepatic enzymes related to cholesterol and bile acid metabolism in rats. Biochim. Biophys. Acta 441, 308-315.

Bémeur, C., Chastre, A., Desjardins, P., Butterworth, R.F., 2010. No changes in expression of tight junction proteins or blood-brain barrier permeability in azoxymethane-induced experimental acute liver failure. Neurochem. Int. 56, 205-207.

Bickel, U., Grave, B., Kang, Y.S., del Rey, A., Voigt, K., 1998. No increase in blood-brain barrier permeability after intraperitoneal injection of endotoxin in the rat. J. Neuroimmunol. $85,131-136$.

Bickel, U., 2005. How to measure drug transport across the blood-brain barrier. NeuroRx 2, 1526.

Bosoi, C.R., Yang, X., Huynh, J., Parent-Robitaille, C., Jiang, W., Tremblay, M., Rose, C.F., 2012. Systemic oxidative stress is implicated in the pathogenesis of brain edema in rats with chronic liver failure. Free Radic. Biol. Med. 52, 1228-1235.

Bowmer, C.J., Donoghue, P.G., Leong, C.F., Yates, M.S., 1985. Effect of bile acids on the binding of drugs and dyes to human albumin. J. Pharm. Pharmacol. 37, 812-815.

Butterworth, R.F., Norenberg, M.D., Felipo, V., Ferenci, P., Albrecht, J., Blei, A.T., 2009. Experimental models of hepatic encephalopathy: ISHEN guidelines. Liver Int. 29, 783-788. 
Cauli, O., Lopez-Larrubia, P., Rodrigo, R., Agusti, A., Boix, J., Nieto-Charques, L., Cerdan, S., Felipo, V., 2011. Brain region-selective mechanisms contribute to the progression of cerebral alterations in acute liver failure in rats. Gastroenterology 140, 638-645.

Chen, F., Radisky, E.S., Das, P., Batra, J., Hata, T., Hori, T., Baine, A.M., Gardner, L., Yue, M.Y., Bu, G., Del Zoppo, G., Patel, T.C., Nguyen, J.H., 2013. TIMP-1 attenuates blood-brain barrier permeability in mice with acute liver failure. J. Cereb. Blood Flow Metab. Epub ahead of print.

Chen, S.C., Nakamura, H., Tamura, Z., 1980. Determination of fluorescein and fluorescein monoglucuronide excreted in urine. Chem. Pharm. Bull. Tokyo 28, 2812-2816.

Coy, D.L., Srivastava, A., Gottstein, J., Butterworth, R.F., Blei, A.T., 1991. Postoperative course after portacaval anastomosis in rats is determined by the portacaval pressure gradient. Am. J. Physiol. 261, G1072-G1078.

Dixit, V., Chang, T.M., 1990. Brain edema and the blood brain barrier in galactosamine-induced fulminant hepatic failure rats. An animal model for evaluation of liver support systems. ASAIO Trans. 36, 21-27.

Ferenci, P., Lockwood, A., Mullen, K., Tarter, R., Weissenborn, K., Blei, A.T., 2002. Hepatic encephalopathy--definition, nomenclature, diagnosis, and quantification: final report of the working party at the 11th World Congresses of Gastroenterology, Vienna, 1998. Hepatology $35,716-721$.

Greenwood, J., Adu, J., Davey, A.J., Abbott, N.J., Bradbury, M.W., 1991. The effect of bile salts on the permeability and ultrastructure of the perfused, energy-depleted, rat blood-brain barrier. J. Cereb. Blood Flow Metab. 11, 644-654. 
Grimes, P.A., 1985. Fluorescein distribution in retinas of normal and diabetic rats. Exp. Eye Res. 41, 227-238.

Hawkins, P.A., DeJoseph, M.R., Vina, J.R., Hawkins, R.A., 1996. Comparison of the metabolic disturbances caused by end-to-side and side-to-side portacaval shunts. J. Appl. Physiol. 80, 885-891.

Horowitz, M.E., Schafer, D.F., Molnar, P., Jones, E.A., Blasberg, R.G., Patlak, C.S., Waggoner, J., Fenstermacher, J.D., 1983. Increased blood-brain transfer in a rabbit model of acute liver failure. Gastroenterology 84, 1003-1011.

Jerkins, A.A., Steele, R.D., 1988. Diet composition and surgical technique influence the postoperative recovery of portacaval shunted rats. Hepatology 8, 855-860.

Jin, S., Wang, X.T., Liu, L., Yao, D., Liu, C., Zhang, M., Guo, H.F., Liu, X.D., 2013. Pglycoprotein and multidrug resistance-associated protein 2 are oppositely altered in brain of rats with thioacetamide-induced acute liver failure. Liver Int. 33, 274-282.

Kang, Y.S., Bickel, U., Pardridge, W.M., 1994. Pharmacokinetics and saturable blood-brain barrier transport of biotin bound to a conjugate of avidin and a monoclonal antibody to the transferrin receptor. Drug Metab. Dispos. 22, 99-105.

Laursen, H., Westergaard, E., 1977. Enhanced permeability to horseradish peroxidase across cerebral vessels in the rat after portacaval anastomosis. Neuropath. Appl. Neurol. Biol. 3, 2943.

Li, W., Rockey, J.H., 1982. Fluorescein binding to normal human serum proteins demonstrated by equilibrium dialysis. Arch. Ophthalmol. 100, 484-487. 
Livingstone, A.S., Potvin, M., Goresky, C.A., Finlayson, M.H., Hinchey, E.J., 1977. Changes in the blood-brain barrier in hepatic coma after hepatectomy in the rat. Gastroenterology 73 , 697-704.

Magide, A.A., Press, C.M., Myant, N.B., Mitropoulos, K.A., Balasubramaniam, S., 1976. The effect of portacaval anastomosis on plasma lipoprotein metabolism in rats. Biochim. Biophys. Acta 441, 302-307.

Manzini, G., Ciana, A., Crescenzi, V., 1979. The interaction of serum albumins with various drugs in aqueous solution. Gel permeation, calorimetric, and fluorescence data. Biophys. Chem. 10, 389-396.

Manzini, G., Crescenzi, V., 1979. On the contemporaneous, reversible interaction of different ligands with serum albumins in dilute aqueous solutions. Fluorescein and phenylbutazone. Biophys. Chem. 10, 397-407.

Mehvar, R., 2005. Role of protein binding in pharmacokinetics. Am. J. Pharm. Educ. 69, article 103.

Mills, C.O., Milkiewicz, P., Muller, M., Roma, M.G., Havinga, R., Coleman, R., Kuipers, F., Jansen, P.L., Elias, E., 1999. Different pathways of canalicular secretion of sulfated and nonsulfated fluorescent bile acids: a study in isolated hepatocyte couplets and TR- rats. J. Hepatol. 31, 678-684.

Nguyen, J.H., 2012. Blood-brain barrier in acute liver failure. Neurochem. Int. 60, 678-683.

Ohno, K., Pettigrew, K.D., Rapoport, S.I., 1978. Lower limits of cerebrovascular permeability to nonelectrolytes in the conscious rat. Am. J. Physiol. 235, H299-H307.

Poupon, R.E., Poupon, R.Y., Grosdemouge, M.L., Erlinger, S., 1977. Effect of portacaval shunt on serum bile acid concentration in patients with cirrhosis. Digestion 16, 138-145. 
Rockey, J.H., Li, W., Eccleston, J.F., 1983. Binding of fluorescein and carboxyfluorescein by human serum proteins: significance of kinetic and equilibrium parameters of association in ocular fluorometric studies. Exp. Eye Res. 37, 455-466.

Roda, A., Cappelleri, G., Aldini, R., Roda, E., Barbara, L., 1982. Quantitative aspects of the interaction of bile acids with human serum albumin. J. Lipid Res. 23, 490-495.

Selan, F., Blair, N., Evans, M.A., 1985. High-performance liquid chromatographic analysis for fluorescein and fluorescein monoglucuronide in plasma. J. Chromatogr. 338, 213-218.

Shimojima, N., Eckman, C.B., McKinney, M., Sevlever, D., Yamamoto, S., Lin, W., Dickson, D.W., Nguyen, J.H., 2008. Altered expression of zonula occludens-2 precedes increased blood-brain barrier permeability in a murine model of fulminant hepatic failure. J. Invest. Surg. 21, 101-108.

Skowronska, M., Zielinska, M., Wojcik-Stanaszek, L., Ruszkiewicz, J., Milatovic, D., Aschner, M., Albrecht, J., 2012. Ammonia increases paracellular permeability of rat brain endothelial cells by a mechanism encompassing oxidative/nitrosative stress and activation of matrix metalloproteinases. J. Neurochem. 121, 125-134.

Spigelman, M.K., Zappulla, R.A., Malis, L.I., Holland, J.F., Goldsmith, S.J., Goldberg, J.D., 1983. Intracarotid dehydrocholate infusion: a new method for prolonged reversible bloodbrain barrier disruption. Neurosurgery 12, 606-612.

Sumner, B.E., 1982. A quantitative study of vascular permeability to horseradish peroxidase, and the subsequent fate of the tracer, in rat brains after portocaval anastomosis. Neuropathol. Appl. Neurobiol. 8, 117-133. 
Sun, H., Johnson, D.R., Finch, R.A., Sartorelli, A.C., Miller, D.W., Elmquist, W.F., $2001 \mathrm{a}$. Transport of fluorescein in MDCKII-MRP1 transfected cells and mrp1-knockout mice. Biochem. Biophys. Res. Commun. 284, 863-869.

Sun, H., Miller, D.W., Elmquist, W.F., 2001b. Effect of probenecid on fluorescein transport in the central nervous system using in vitro and in vivo models. Pharm. Res. 18, 1542-1549.

Zaki, A.E., Wardle, E.N., Canalese, J., Ede, R.J., Williams, R., 1983. Potential toxins of acute liver failure and their effects on blood-brain barrier permeability. Experientia 39, 988-991.

Zaki, A.E., Ede, R.J., Davis, M., Williams, R., 1984. Experimental studies of blood brain barrier permeability in acute hepatic failure. Hepatology 4, 359-363.

Ziylan, Y.Z., Uzum, G., Bernard, G., Diler, A.S., Bourre, J.M., 1993. Changes in the permeability of the blood-brain barrier in acute hyperammonemia. Effect of dexamethasone. Mol. Chem. Neuropathol. 20, 203-218. 


\section{Figure Legends}

Fig. 1 - Liver: total body weight ratio (a) and plasma concentrations of ammonia (b), total bile acids (c), total proteins (d), albumin (e), and AST (f) in Sham ( $n=6)$ and PCA ( $n=5)$ animals. Data are collected 10 days after PCA or sham surgery. The symbols and horizontal lines represent the individual and mean values, respectively. Statistical analysis is based on unpaired, two-tailed, t-test.

Fig. 2 - Total (free plus bound to plasma proteins) (a) and free (b) plasma concentration-time courses and plasma free fraction $\left(f_{u}\right)$-time courses (c) of fluorescein in rats 10 days after sham (n =6) or PCA ( $n=5)$ surgery. A single $25 \mathrm{mg} / \mathrm{kg}$ dose of fluorescein was administered intravenously through a 5 min constant infusion 10 days after the surgery. The symbols and bars represent the mean and SD values, respectively. Statistical analysis is based on repeated measure, two-way ANOVA, followed by Bonferroni's multiple comparison test. ${ }^{*} p<0.05,{ }^{* *} p<0.01$.

Fig. 3 - Biliary recovery (a), biliary clearance of total (free plus bound) (b) and free (unbound) (c) fluorescein, biliary recovery of fluorescein-glucuronide (d), overall (fluorescein plus fluorescein glucuronide) biliary recovery (e), and bile flow rate (f) in Sham (n=6) and PCA ( $n=5)$ animals. A single $25 \mathrm{mg} / \mathrm{kg}$ dose of fluorescein was administered intravenously through a $5 \mathrm{~min}$ constant infusion 10 days after the surgery, and samples were collected for $30 \mathrm{~min}$. The symbols and horizontal lines represent the individual and mean values, respectively. Statistical analysis is based on unpaired, two-tailed, t-test.

Fig. 4 - The concentration (a) and amount (b) of fluorescein recovered in the liver in Sham ( $n=6)$ and PCA ( $n=5)$ animals. A single $25 \mathrm{mg} / \mathrm{kg}$ dose of fluorescein was administered intravenously through a 5 min constant infusion 10 days after the surgery, and liver samples were collected 
after $30 \mathrm{~min}$. The symbols and horizontal lines represent the individual and mean values, respectively. Statistical analysis is based on unpaired, two-tailed, t-test.

Fig. 5 - The brain concentration (a) and total (b) and free (c) brain uptake clearance $\left(K_{i n}\right)$ of fluorescein in Sham $(n=6)$ and PCA $(n=5)$ animals. A single $25 \mathrm{mg} / \mathrm{kg}$ dose of fluorescein was administered intravenously through a 5 min constant infusion 10 days after the surgery, and brain samples were collected after $30 \mathrm{~min}$. The symbols and horizontal lines represent the individual and mean values, respectively.

Fig. 6 - The effects of different concentrations of lithocholic acid (LCA), cholic acid (CA), chenodeoxycholic acid (CDCA), and taurocholic acid (TCA) on the in vitro free fraction of fluorescein in rat plasma $(n=3)$. The concentration of fluorescein in the plasma samples was 50 $\mu \mathrm{g} / \mathrm{ml}$. The symbols and bars represent the mean and SD values, respectively. 
(a)

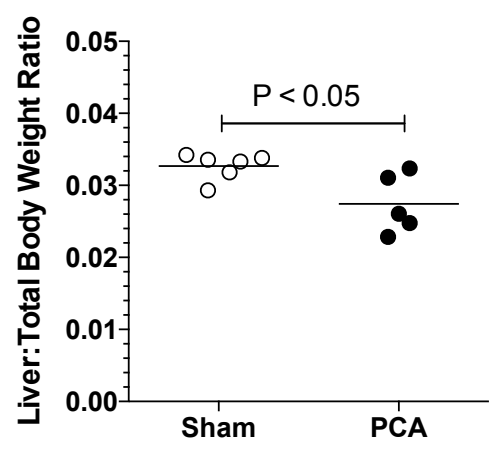

(d)

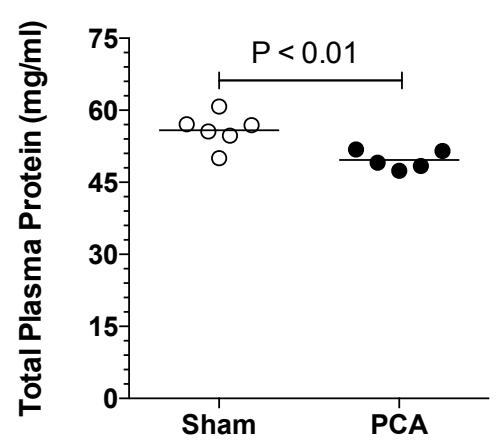

(b)

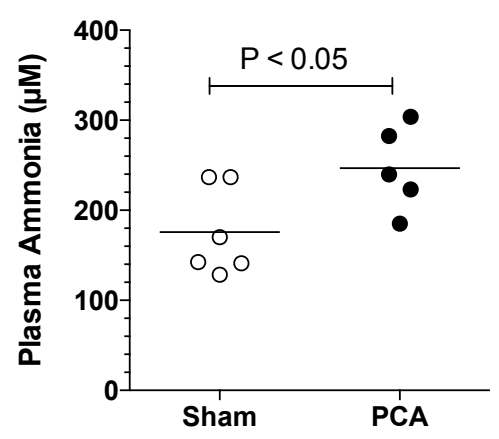

(e)

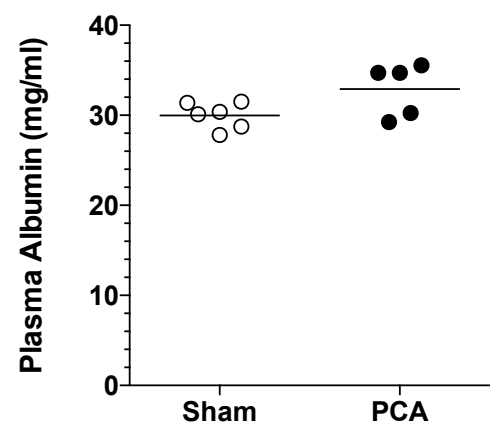

(c)

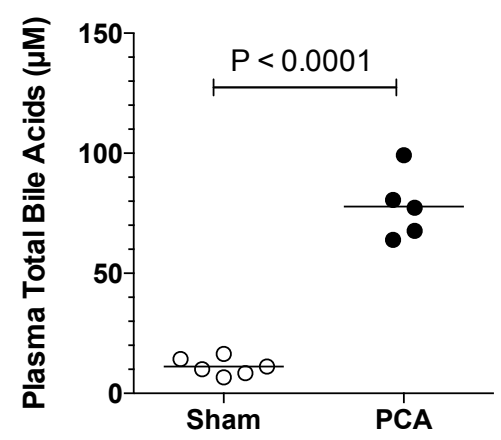

(f)

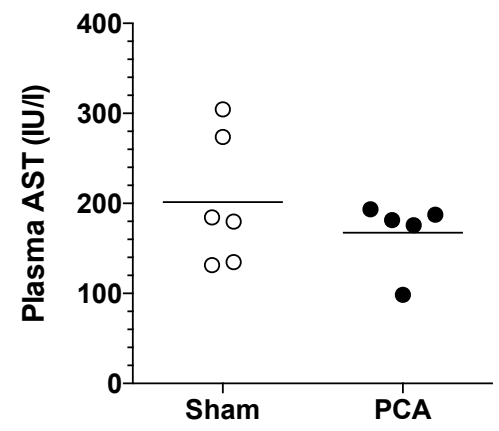


(a)

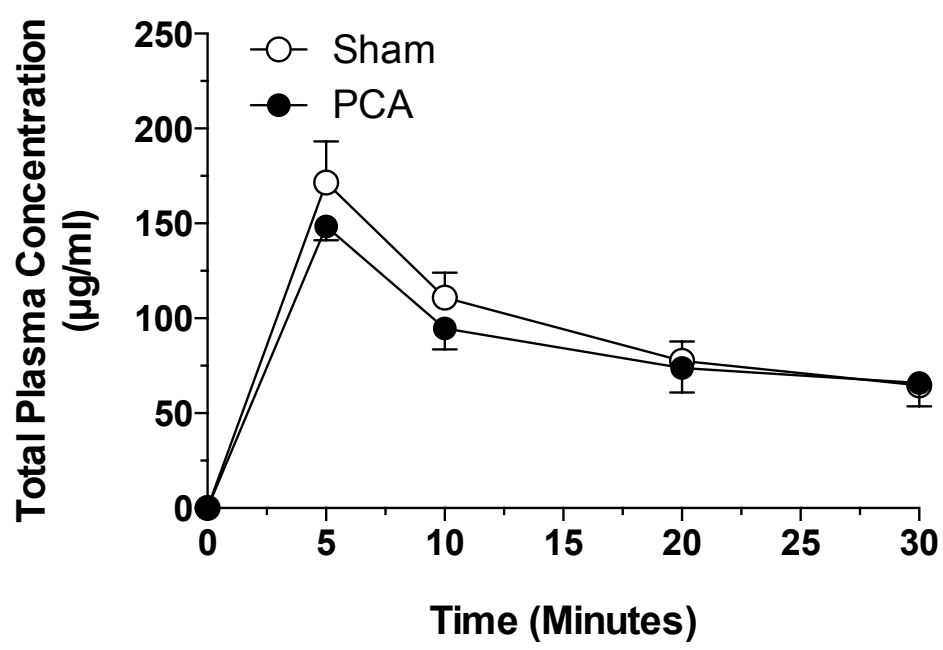

(b)

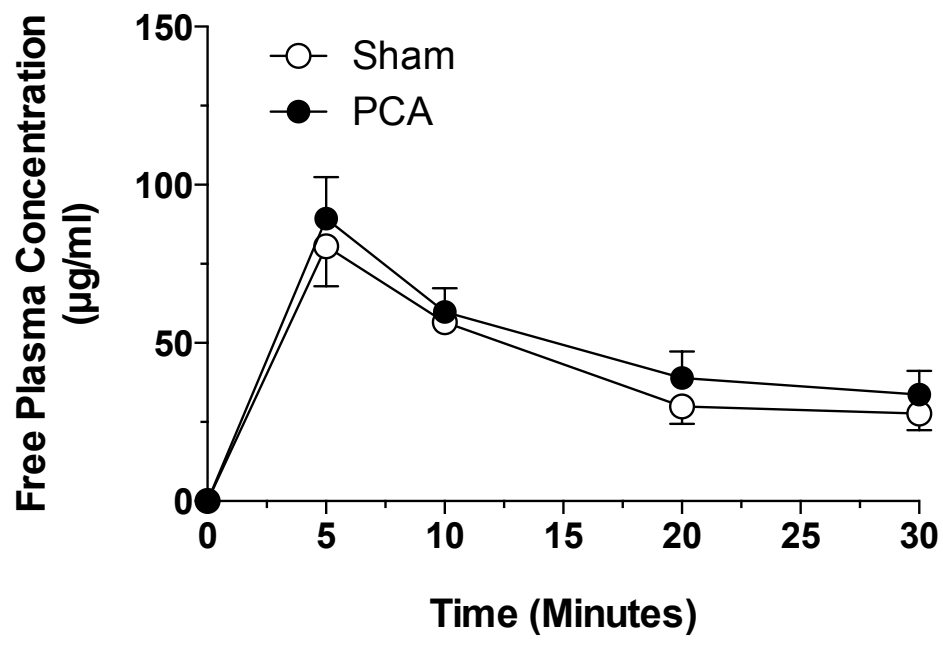

(c)

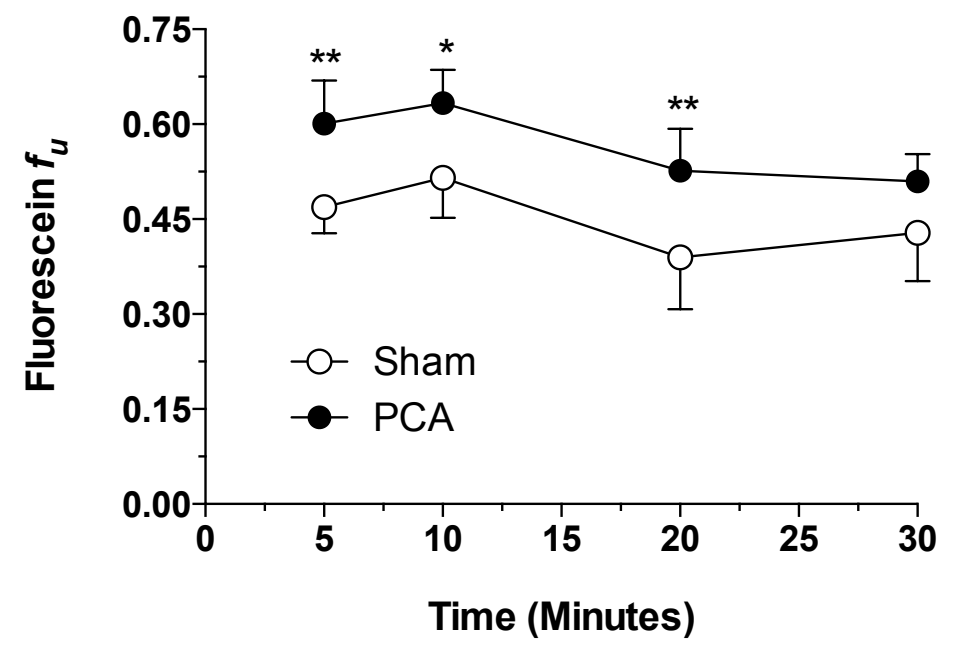


(a)

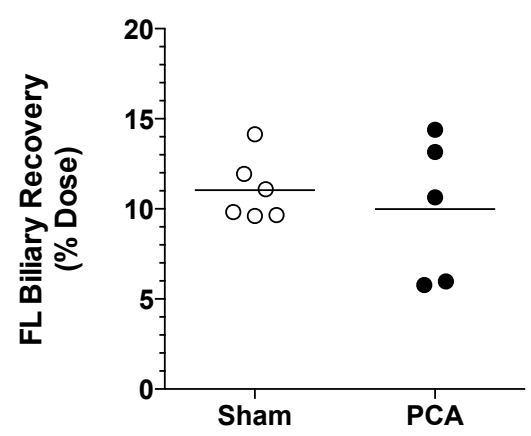

(d)

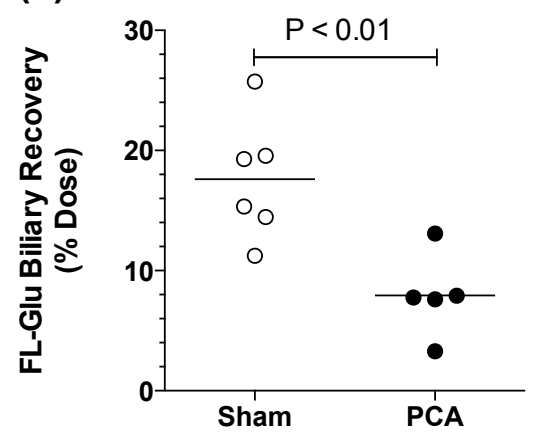

(b)

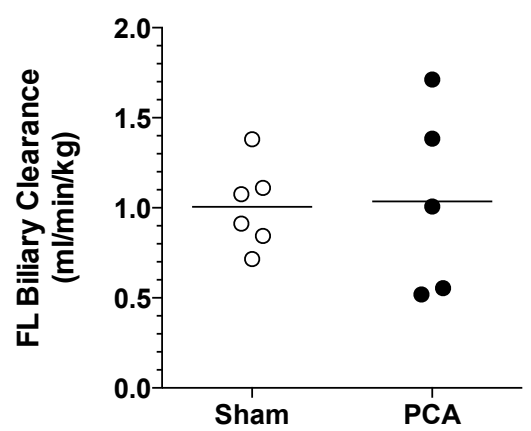

(e)

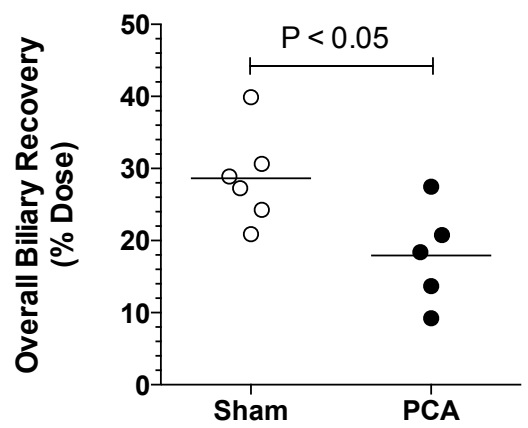

(c)

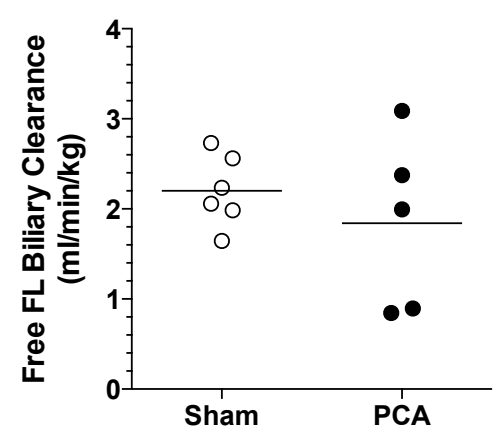

(f)

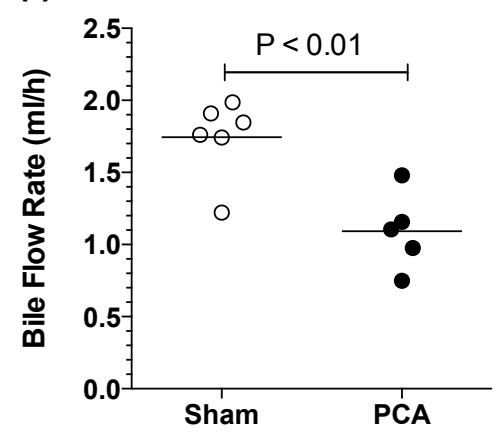


Figure 4

(a)

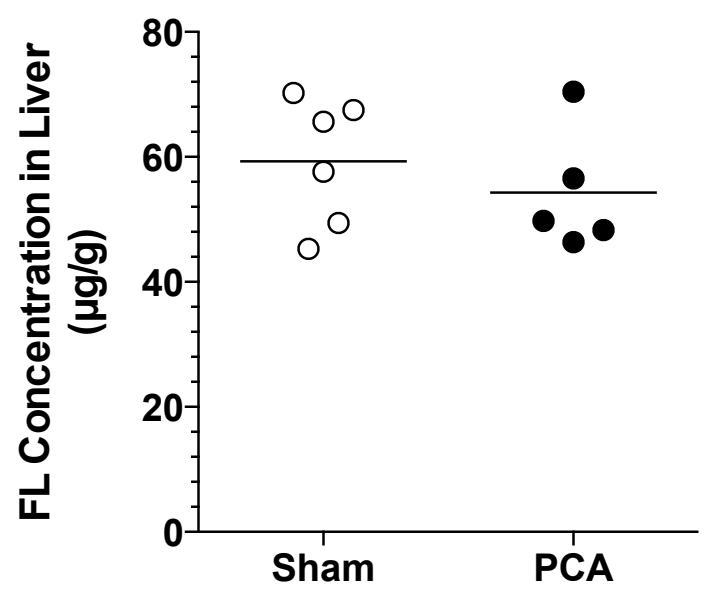

(b)

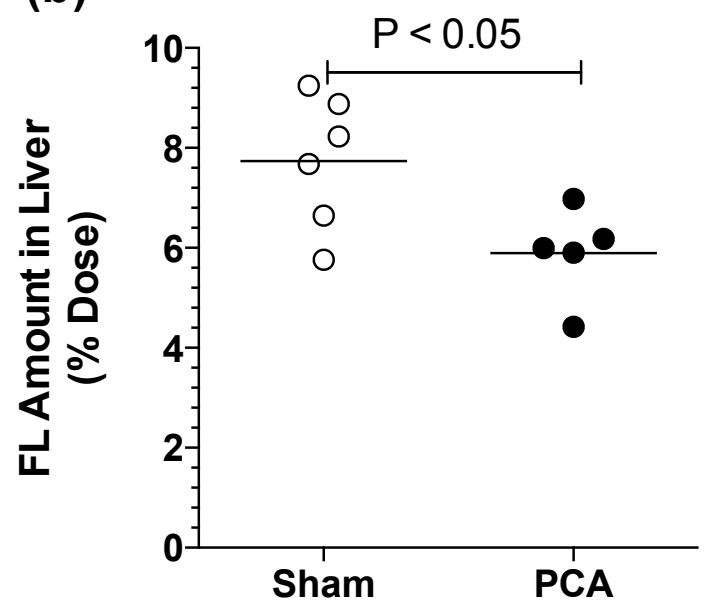


(a)

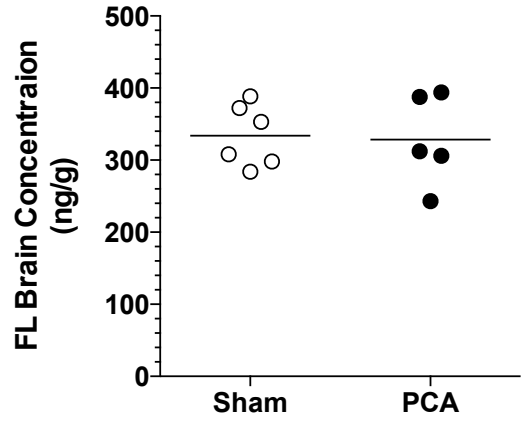

(b)

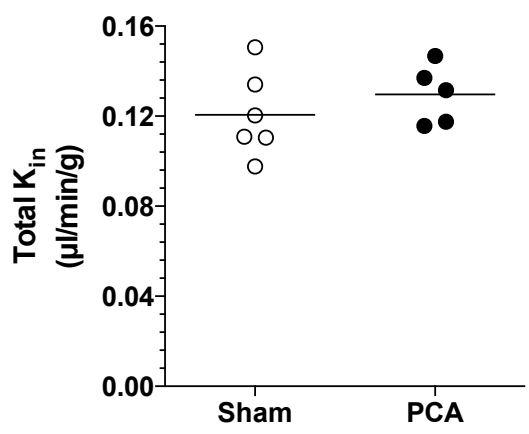

(c)

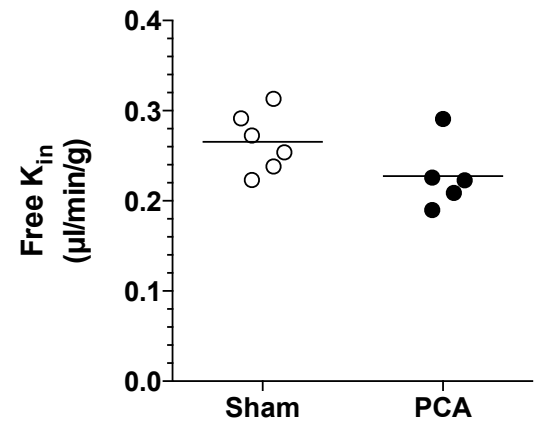




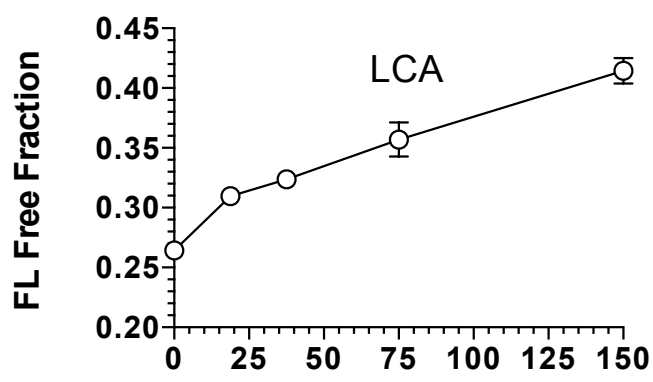

Bile Acid Concentrtaion ( $\mu \mathrm{M})$

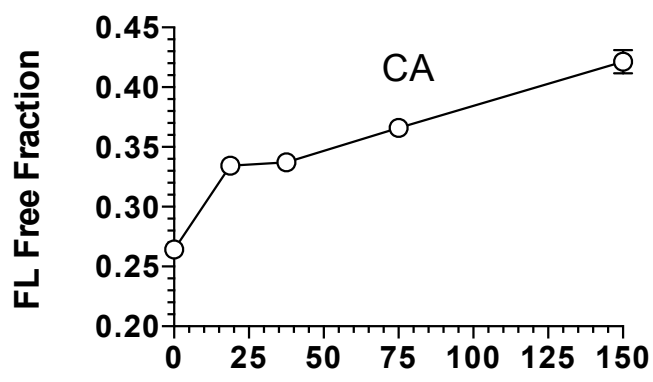

Bile Acid Concentrtaion ( $\mu \mathrm{M})$

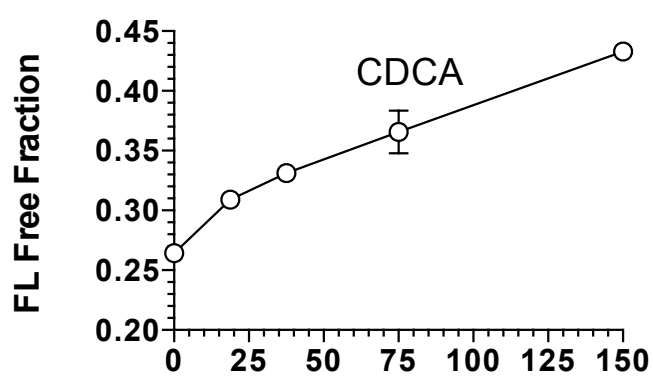

Bile Acid Concentrtaion ( $\mu \mathrm{M})$

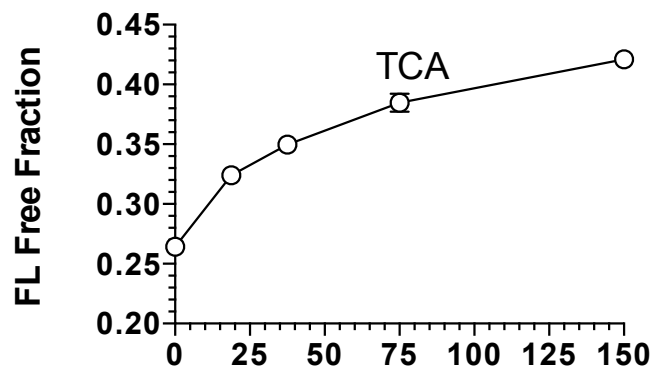

Bile Acid Concentrtaion $(\mu \mathrm{M})$ 
Table 1 - Effects of portacaval anastomosis (PCA) on the area under the plasma concentration-time curves of total (free plus protein-bound, $A U C_{t o t a l}$ ) and free (unbound, $A U C_{\text {free }}$ ) fluorescein and the free: total ratios.

\begin{tabular}{llll}
\hline & $A U C_{\text {total }}$ & $A U C_{\text {free }}$ & $A U C_{\text {free }}: A U C_{\text {total }}$ \\
\hline Sham & $2790 \pm 311$ & $1260 \pm 136$ & $0.455 \pm 0.050$ \\
PCA & $2520 \pm 293$ & $1453 \pm 238$ & $0.575 \pm 0.047$ \\
PCA: Sham & 0.903 & 1.15 & 1.26 \\
$p$ & NS & NS & $<0.01$ \\
\hline A & of & fluorescein was administered intravenously through a 5 min constant
\end{tabular}

A single $25 \mathrm{mg} / \mathrm{kg}$ dose of fluorescein was administered intravenously through a 5 min constant infusion 10 days after sham $(n=6)$ or PCA $(n=5)$ surgery, and plasma concentrations were collected over $30 \mathrm{~min}$. The data are presented as mean \pm SD. Statistical analysis is based on unpaired, two-tailed, t-test (NS: not significant). 